\title{
O carbonato de cálcio na desacidificação do vinho isabel
}

\author{
The calcium carbonate in the desacidification of isabella wine
}

Luiz Antenor Rizzon ${ }^{1}$ André Miguel Gasparin²

\section{RESUMO}

A uva Isabel (Vitis labrusca) é a cultivar de videira mais difundida na Região Vitícola da Serra Gaúcha. Entre outras finalidades, é utilizada para a elaboração de vinho tinto de mesa, o qual, geralmente, apresenta acidez elevada, devido ao teor de ácido tartárico livre. O objetivo do presente trabalho foi avaliar a influência de diferentes doses de carbonato de cálcio $(0,0 ; 0,5 ; 1,0 ; 1,5 ; 2,0 ; 2,5 e$ 3,0 $\mathrm{g} \mathrm{L}^{-1}$ ) na correção da acidez e na composição do vinho Isabel da Serra Gaúcha. O estudo foi realizado na Embrapa Uva e Vinho, em Bento Gonçalves - RS, na safra de 2002. O delineamento experimental utilizado foi o de blocos casualizados, com sete tratamentos e quatro repetições. As análises dos vinhos, realizadas dez dias após o tratamento, constaram da densidade, álcool, acidez total, acidez volátil, $\mathrm{pH}$, açúcares redutores, extrato seco, extrato seco reduzido, cinzas, densidade ótica a 420, 520 e $620 \mathrm{~nm}$, intensidade de cor e coloração, efetuadas através de métodos físico-químicos. O ácido tartárico foi determinado através da cromatografia líquida de alta eficiência (CLAE). O potássio $e$ o cálcio foram analisados por espectrofotometria de absorção atômica. Além da redução da acidez do vinho Isabel, o carbonato de cálcio interferiu na cor, no extrato seco, nas cinzas e no teor de elementos minerais do vinho Isabel.

Palavras-chave: Vitis labrusca, desacidificação, composição química.

\section{ABSTRACT}

Isabel grape (Vitis labrusca) is the variety mostly spread in the Serra Gaúcha Region which is used, among other purposes, to elaborate red table wines. This wine usually presents high acidity, due to the level of free tartaric acid. The purpose of this work was to evaluate the effect of different doses of calcium carbonate in acidity and in the Isabel wine composition of the Serra Gaúcha region. The study carried out at Embrapa Uva e Vinho consisted of application in Isabel wine, from the 2002 vintage, different concentrations of calcium carbonate $(0,0 ; 0,5 ; 1,0 ; 1,5 ; 2,0 ; 2,5$ and $3,0 \mathrm{~g} \mathrm{~L}$ $\left.{ }^{1}\right)$. The experimental design was a randomized block with seven treatments and four replications. The wines were analyzed ten days after treatment. The determinations were accomplished by physical-chemical methods: density, alcohol, total titratable acidity, $\mathrm{pH}$, reducing sugar, dry extract, reduced dry extract, ashes, optical density at 420, 520 and $620 \mathrm{~nm}$, color intensity and coloration. The tartaric acid was measured by liquid chromatography. The potassium and calcium were analyzed by atomic absorption spectrometry. Results showed that besides the reduction of acidity, the calcium carbonate interfered in color, dry extract, ashes and in the mineral elements of the Isabel wine.

Key words: Vitis labrusca, desacidification, chemical composition.

Na Serra Gaúcha, região vitivinícola mais importante do Brasil, predomina o vinho de mesa da cultivar Isabel (Vitis labrusca), o qual possui acidez elevada (RIZZON et al., 2000; RIZZON \& MIELE, 2002). A acidez do vinho é avaliada através da acidez total, do pH e da concentração individual dos princi-

${ }^{1}$ Engenheiro Agrônomo, Pesquisador, Embrapa Uva e Vinho, CP 130, 95700-000, Bento Gonçalves, RS, Brasil. E-mail: rizzon@cnpuv.embrapa.br. Autor para correspondência.

${ }^{2}$ Estudante do Curso de Tecnologia em Viticultura e Enologia, Centro Federal de Educação Tecnológica (CEFET), 95700-000 Bento Gonçalves, RS, Brasil. Bolsista de Iniciação Científica da Fundação de Amparo à Pesquisa do Estado do Rio Grande do Sul (FAPERGS). 
pais ácidos. As alternativas enológicas para reduzir a acidez baseiam-se na realização da fermentação malolática, em favorecer a salificação dos ácidos através de macerações mais longas e na precipitação do ácido tartárico (BOULTON et al., 1995). A desacidificação química neutraliza o excesso de acidez e precipita os sais formados. Os sais precipitáveis na desacidificação são o tartarato de cálcio, o bitartarato de potássio e em condições especificas o sal duplo, tartarato e malato de cálcio (USSEGLIOTOMASSET, 1995). Os produtos enológicos mais utilizados para a desacidificação dos vinhos são o carbonato de cálcio, o bicarbonato de potássio e o carbonato de potássio (RIBÉREAU-GAYON et al., 1998).

No vinho Isabel, a acidez é devida ao teor elevado de ácido tartárico livre e ao baixo teor de potássio (RIZZON et al., 2000; RIZZON \& MIELE, 2002), por isso, é mais indicado utilizar produtos enológicos que reduzem esse ácido, no entanto, o seu teor não pode ser inferior a $1,0 \mathrm{~g} \mathrm{~L}^{-1}$ (OFFICE INTERNATIONAL DE LA VIGNE ET DU VIN, 1990). Nesse sentido, o objetivo do presente trabalho foi avaliar o efeito de diferentes doses de carbonato de cálcio na redução da acidez e na composição do vinho Isabel da Serra Gaúcha.

O trabalho foi realizado na Embrapa Uva e Vinho, localizada em Bento Gonçalves, RS, com vinho Isabel, safra 2002, com $128 \mathrm{meq} \mathrm{L}^{-1}$ de acidez total, 9meq $\mathrm{L}^{-1}$ de acidez volátil e pH de 2,95. Utilizou-se carbonato de cálcio comercial de uso enológico o qual foi aplicado nas proporções de $(0,0 ; 0,5 ; 1,0 ; 1,5 ; 2,0 ; 2,5$ e $\left.3,0 \mathrm{~g} \mathrm{~L}^{-1}\right)$, num delineamento experimental completamente casualizado, com sete tratamentos e quatro repetições. Um volume de 84L de vinho foi homogeneizado e distribuído em 28 recipientes de vidro de 3,0L, que constituíram as unidades experimentais. $\mathrm{O}$ carbonato de cálcio foi dissolvido em um pouco de vinho e adicionado no recipiente. A seguir, o vinho foi homogeneizado e mantido em repouso por um período de dez dias à temperatura ambiente. Após este período, o vinho foi engarrafado e analisado.

A densidade foi determinada através de densímetro digital Anton Paar DMA 45. O álcool foi analisado através da destilação, com um aparelho Gibertini e posterior medida por densimetria (OFFICE INTERNATIONAL DE LA VIGNE ET DU VIN, 1990). A acidez total foi avaliada por volumetria, e a acidez volátil, através da separação dos ácidos voláteis pelo arraste de vapor em um aparelho Cazenave Ferré. O $\mathrm{pH}$ foi determinado através de um $\mathrm{pH}$-metro calibrado com soluções tampão de pH 3,00 e 4,00 (RIBÉREAUGAYON et al., 1998). Os açúcares redutores foram analisados pela reação entre a solução cupro-alcalina e os açúcares presentes na amostra e o excesso de íons cúpricos determinados por iodometria. O extrato seco foi determinado pelo método direto com um banhomaria. O extrato seco reduzido corresponde ao extrato seco descontado da quantidade de açúcar superior a $1,0 \mathrm{~g} \mathrm{~L}^{-1}$ (OFFICE INTERNATIONAL DE LA VIGNE ET DU VIN, 1990). As cinzas foram obtidas através da incineração à temperatura de $550^{\circ} \mathrm{C}$ de uma alíquota de vinho. Os índices de cor DO 420; DO 520 e DO $620 \mathrm{~nm}$ foram determinados através da leitura espectrofotométrica, com uma cubeta de quartzo de 1 mm de percurso ótico. A intensidade de cor representa a soma das leituras obtidas. A coloração representa o valor da relação entre a DO 420 e a DO 520nm (RIBÉREAU-GAYON et al., 1998). O ácido tartárico foi analisado através de cromatografia líquida de alta eficiência (CLAE), utilizando-se um injetor Rheodyne de 20mL e uma coluna Varian MCH-NCAP-5 de 4mm x $15 \mathrm{~cm}$. O detector foi fixado em um comprimento de onda de 212nm. Na eluição, utilizou-se um solvente constituído de água ultrapura acidificada com ácido fosfórico a pH 2,5 (AUGUSTE, 1979). O potássio foi analisado através da emissão de chama, enquanto o cálcio, por absorção atômica, em um espectrofotômetro de absorção atômica marca Perkin-Elmer modelo 2380 (PERKIN-ELMER, 2000). Os resultados obtidos foram submetidos ao teste de Tukey, para comparação das médias, a 5\% de probabilidade de erro e à determinação do coeficiente de correlação entre os níveis de carbonato de cálcio e as variáveis.

Constatou-se que, além das variáveis relacionadas à acidez tais como: acidez total, pH e ácido tartárico, o carbonato de cálcio modificou a densidade, o extrato, as cinzas, a coloração e os elementos minerais do vinho Isabel (Tabela 1). A ação do carbonato de cálcio na redução da acidez total, e conseqüente aumento do valor do $\mathrm{pH}$, foi devido à salificação do ácido tartárico, insolubilização e precipitação do tartarato de cálcio (GABAS et al., 1994; USSEGLIO-TOMASSET, 1995; RIBÉREAU-GAYON et al., 1998; BLOUIN, 2002). A quantidade de carbonato de cálcio a ser utilizada não deve ser excessiva pois deve permanecer sempre no vinho Isabel, no mínimo 2,0g L-1 de ácido tartárico, para manter as suas características de frescor. Nesse sentido, recomenda-se não aplicar doses excessivas de carbonato de cálcio nos vinhos, pois, além de descaracterizá-los, causam problemas de turvação devido ao excesso de cálcio, que geralmente ocorrem no vinho engarrafado. Em relação à densidade do vinho Isabel, observou-se que o carbonato de cálcio provocou uma redução nos valores, em conseqüência da precipitação do ácido tartárico o qual foi responsável também pela diminuição do teor de extrato seco e do extrato 
Tabela 1 - Influência do Carbonato de Cálcio na desacidificação e na composição do vinho Isabel.

\begin{tabular}{|c|c|c|c|c|c|c|c|c|c|c|}
\hline \multirow{2}{*}{ Variáveis } & \multicolumn{7}{|c|}{ Carbonato de Cálcio (g L L ${ }^{-1} *$} & \multirow[t]{2}{*}{ Média } & \multirow[t]{2}{*}{$\mathrm{CV}$} & \multirow{2}{*}{$\begin{array}{l}\text { Coeficiente de } \\
\text { correlação (r) }\end{array}$} \\
\hline & 0 & 0,5 & 1,0 & 1,5 & 2,0 & 2,5 & 3,0 & & & \\
\hline Densidade a $20^{\circ} \mathrm{C}\left(\mathrm{g} \mathrm{mL}^{-1}\right)$ & $0,9952 \mathrm{a}$ & $0,9948 \mathrm{~b}$ & $0,9946 \mathrm{c}$ & $0,9943 \mathrm{~d}$ & $0,9942 \mathrm{e}$ & $0,9942 \mathrm{e}$ & $0,9943 \mathrm{de}$ & 0,9945 & 0,0006 & $-0,8821$ \\
\hline Álcool (\% v/v) & 11,39 a & $11,40 \mathrm{a}$ & 11,45 a & 11,41 a & $11,41 \mathrm{a}$ & $11,42 \mathrm{a}$ & $11,43 \mathrm{a}$ & 11,42 & 0,35 & -0.4657 \\
\hline Acidez Total (meq L $\left.{ }^{-1}\right)$ & $127,2 \mathrm{a}$ & $122,4 \mathrm{~b}$ & 116,2 c & $109,4 \mathrm{~d}$ & $96,3 \mathrm{e}$ & $93,3 \mathrm{f}$ & $79,3 \mathrm{~g}$ & 107,0 & 1,58 & 0,9875 \\
\hline Acidez Volátil (meq L ${ }^{-1}$ ) & $9,7 \mathrm{a}$ & $10,0 \mathrm{a}$ & 10,0 a & $10,1 \mathrm{a}$ & $10,1 \mathrm{a}$ & $10,2 \mathrm{a}$ & $10,8 \mathrm{a}$ & 10,1 & 9,49 & $-0,8745$ \\
\hline $\mathrm{pH}$ & $2,95 \mathrm{~g}$ & $3,00 \mathrm{f}$ & $3,07 \mathrm{e}$ & $3,17 \mathrm{~d}$ & $3,34 \mathrm{c}$ & $3,52 b$ & $3,73 \mathrm{a}$ & 3,25 & 0,25 & 0,9730 \\
\hline Açúcares Redutores (g L-1) & $2,95 \mathrm{a}$ & $2,93 \mathrm{a}$ & $2,99 \mathrm{a}$ & $2,95 \mathrm{a}$ & $2,95 \mathrm{a}$ & $2,94 \mathrm{a}$ & $2,94 \mathrm{a}$ & 2,95 & 1,31 & 0,2015 \\
\hline Extrato Seco $\left(\mathrm{g} \mathrm{L}^{-1}\right)$ & 26,6 a & $25,7 \mathrm{~b}$ & $25,2 \mathrm{c}$ & $24,5 d$ & $24,1 \mathrm{~d}$ & $24,1 \mathrm{~d}$ & $24,4 \mathrm{~d}$ & 24,9 & 0,79 & 0,8950 \\
\hline Extrato Seco reduzido $\left(\mathrm{g} \mathrm{L}^{-1}\right)$ & 23,6 a & $22,7 \mathrm{~b}$ & $22,2 \mathrm{c}$ & $21,6 \mathrm{~d}$ & $21,1 \mathrm{e}$ & $21,1 \mathrm{e}$ & 21,4 de & 22,0 & 0,83 & 0,9018 \\
\hline Cinzas $\left(\mathrm{g} \mathrm{L}^{-1}\right)$ & 2,32 bc & $2,23 \mathrm{c}$ & 2,31 bc & 2,33 bc & $2,38 \mathrm{~b}$ & $2,45 b$ & $2,82 \mathrm{a}$ & 2,41 & 2,52 & 0,7966 \\
\hline DO 420 (nm) & 0,232 a & 0,231 a & $0,222 \mathrm{~b}$ & 0,212 c & $0,204 \mathrm{~d}$ & 0,195 e & $0,198 \mathrm{f}$ & 0,212 & 1,15 & 0,9712 \\
\hline DO 520 (nm) & $0,502 \mathrm{a}$ & 0,496 a & $0,460 \mathrm{~b}$ & 0,417 c & $0,379 \mathrm{~d}$ & 0,329 e & $0,292 \mathrm{f}$ & 0,411 & 2,11 & 0,9893 \\
\hline DO $620(\mathrm{~nm})$ & 0,063 bc & $0,063 \mathrm{bc}$ & 0,062 bc & 0,062 c & 0,064 bc & $0,065 \mathrm{~b}$ & 0,069 a & 0,064 & 1,79 & 0,7559 \\
\hline $\begin{array}{l}\text { Intensidade de Cor } \\
(\mathrm{DO} 420+\mathrm{DO} 520+\mathrm{DO} 620)\end{array}$ & 0,798 a & 0,791 a & $0,745 \mathrm{~b}$ & 0,681 c & $0,648 \mathrm{~d}$ & 0,589 e & $0,550 \mathrm{f}$ & 0,686 & 2,01 & 0,9905 \\
\hline Coloração (DO 420/DO 520) & $0,465 \mathrm{ab}$ & $0,467 \mathrm{ab}$ & $0,372 \mathrm{~b}$ & $0,510 \mathrm{ab}$ & $0,540 \mathrm{ab}$ & $0,590 \mathrm{a}$ & $0,647 \mathrm{a}$ & 0,513 & 15,89 & 0,8202 \\
\hline Ácido Tartárico (g L $\left.{ }^{-1}\right)$ & $4,12 \mathrm{a}$ & $3,23 \mathrm{~b}$ & $2,70 \mathrm{c}$ & $1,90 \mathrm{~d}$ & $1,30 \mathrm{e}$ & $0,80 \mathrm{f}$ & 0,88 ef & 2,14 & 9,86 & 0,9763 \\
\hline Potássio (mg L ${ }^{-1}$ ) & 930,0 a & 934,3 a & $957,4 \mathrm{a}$ & 958,6 a & 957,4 a & 935,9 a & 953,9 a & 946,8 & 1,5 & 0,4541 \\
\hline Cálcio (mg L $\left.{ }^{-1}\right)$ & $113,2 \mathrm{~d}$ & $103,1 \mathrm{e}$ & $97,1 \mathrm{e}$ & 102,3 e & 139,9 c & 204,1 b & 333,6 a & 156,2 & 2,8 & 0,8063 \\
\hline
\end{tabular}

* Médias seguidas por letras distintas na linha diferem entre si pelo teste de Tukey, a 5\% de probabilidade de erro.

seco reduzido do vinho Isabel (Tabela 1). Quanto às cinzas do vinho Isabel, detectou-se aumento no teor, nas doses de carbonato de cálcio superiores a 2,0 $\mathrm{g} \mathrm{L}^{-1}$; no entanto, somente com a aplicação de $3,0 \mathrm{~g} \mathrm{~L}^{-1}$ de carbonato de cálcio observou-se diferença significativa, devido ao excesso de cálcio.

Os resultados obtidos não mostraram diferenças significativas para o álcool, acidez volátil, açúcares redutores e potássio em função da concentração de carbonato de cálcio aplicado.

Em relação aos índices de cor (DO 420, DO 520 e DO 620nm), constatou-se que o carbonato de cálcio foi responsável por uma redução da cor amarela do vinho, DO 420nm, assim como, por uma redução da cor vermelha, DO 520nm, e por um aumento da cor azul, DO 620nm, nesse caso, somente na concentração mais alta de carbonato de cálcio. Quanto à coloração, observou-se diferença estatística entre o vinho que foi adicionado de $1,0 \mathrm{~g} \mathrm{~L}^{-1}$ de carbonato de cálcio, que apresentou o valor mais baixo, portanto, com uma predominância da tonalidade vermelha, e o vinho ao qual foram adicionados 3,0g L $\mathrm{g}^{-1}$ de carbonato de cálcio, com valor mais elevado, predominando a cor amarela, provavelmente devido à alteração do $\mathrm{pH}$ que possibilitou a oxidação do mesmo.

Em relação ao cálcio, inicialmente, observou-se redução do seu teor até o tratamento de 2,0g L $\mathrm{g}^{-1}$ de carbonato de cálcio. Nesses vinhos, prevaleceu a precipitação do tartarato de cálcio. Nos tratamentos com 2,0; 2,5 e 3,0 $\mathrm{g} \mathrm{L}^{-1}$ de carbonato de cálcio, constatou-se um incremento acentuado de cálcio nos vinhos, causando problema de estabilidade aos mesmos, pois a precipitação dos sais de cálcio é lenta e, geralmente, ocorre depois do engarrafamento. A utilização do carbonato de cálcio no vinho Isabel da Serra Gaúcha deve ser feita depois de concluída a fermentação malolática com precaução em relação à estabilização e à precipitação do tartarato de cálcio. Além da redução da acidez, o carbonato de cálcio interferiu na cor, no extrato seco, nas cinzas e no teor de elementos minerais do vinho Isabel. Teores elevados de carbonato de cálcio determinam concentrações excessivas de cálcio, provocando problemas de estabilização.

\section{REFERÊNCIAS BIBLIOGRÁFICAS}

AUGUSTE, M.H. Application de la chromatographie em phase liquide à haute pression à l'analyse des moûts et des vins. 1979. 135f. Tese (Doutorado em EnologiaAmepelologia) - Université de Bordeaux II, Talence.

BLOUIN, J. Connaissance des acidités des moûts et vins. Revue des Oenologues et des techniques vitivinicoles et oenologiques, Chaintré, v.29, n.105, p.9-10, 2002.

BOUlton, R.B. et al. Principles and practices of winemaking. New York : Chapman \& Hall, 1995. 604p. 
GABAS, N. et al. Les sels tartriques dans les vins: solubilité et sursaturation. In: LALLEMAND. La microbiologie des vins mousseaux. La stabilisation des vins. Mecanismes et evaluation. Toulouse : Danona, 1994. p.95-98.

OFFICE INTERNATIONAL DE LA VIGNE ET DU VIN. Recueil des méthodes internationales d'analyse des vins. Paris : OIV, 1990. 368p.

PERKIN-ELMER. Analytical methods for atomic absorption spectrometry. Singapura: Perkin-Elmer Instruments, 2000. 300p.
RIBÉREAU-GAYON, P. et al. Traité d'oenologie. 2. Chimie du vin stabilisation et traitements. Paris : Dunod, 1998. 519p.

RIZZON, L.A. et al. Avaliação da cv. Isabel para a elaboração de vinho tinto. Ciência e Tecnologia de Alimentos, Campinas, v.20, n.1, p.115-121, 2000.

RIZZON, L.A.; MIELE, A. Acidez na vinificação em tinto das uvas Isabel, Cabernet Sauvignon e Cabernet Franc. Ciência Rural, Santa Maria, v.32, n.3, p.511-515, 2002.

USSEGLIO-TOMASSET, L. Chimica enologica. Brescia : AEB, 1995. 431p. 